\title{
Afrontamiento ante el impacto del desempleo de larga duración en Bizkaia: relatos de incertidumbre e inseguridad
}

\author{
Ainhoa Díez Sanz \\ Equipo de Investigación Deusto Valores Sociales, Universidad de Deusto \\ <ainhoadiez@deusto.es>
}

\section{Iratxe Aristegui Fradua}

Departamento de Trabajo Social y Sociología, Universidad de Deusto

<iariste@deusto.es>

\section{María Silvestre Cabrera}

Departamento de Trabajo Social y Sociología, Universidad de Deusto

$<$ maria.silvestre@deusto.es>

Artikulu honek, Bizkaiko testuinguruan, semealabak kargura dituzten familiek epe luzeko langabeziari nola aurre egiten dieten ezagutaraztea du helburu. Horretarako, ikerketa kualitatibo bat burutu da 23 pertsona langabeturi egindako sakoneko elkarrizketen bitartez. Ikerketak erakusten du 'baliabide ekonomikoetara sarrera' eta 'babes sozialaren eskuragarritasuna' kategoriek, familiek beraien egoeraz egiten duten balorazioan, etorkizunaren inguruan egiten dituzten aurreikuspenetan eta nahietan zuzenean eragiten dutela eta, hortaz, beraien egoera sozioekonomikoa mantentzeko ala hobetzeko erabiltzen dituzten estrategietan ere eragiten dutela. Bi kategoria hauek ardatz egituratzaile bezala erabiliz, kalteberatasun egoeran dauden familiek langabeziaren eraginari nola aurre egiten dioten azaltzen duen eredu esplikatibo baten eraikitzea aurkezten da.
Este artículo pretende dar a conocer las estrategias de afrontamiento que emplean las familias con prole a cargo de cara a hacer frente al desempleo de larga duración en el contexto vizcaíno. Para ello, se ha llevado a cabo un estudio de carácter cualitativo por medio de entrevistas en profundidad y con una muestra de 23 personas desempleadas. El estudio concluye que el 'acceso a recursos económicos' y la 'disponibilidad de apoyo social' emergen como categorías que determinan la valoración que realizan las familias de su propia situación, sus expectativas $y$ aspiraciones futuras, y que, por tanto, también condicionan las estrategias de afrontamiento que emplean para mantener o mejorar su situación socioeconómica. Tomando como ejes vertebradores ambas categorías, se presenta la construcción de un modelo explicativo del afrontamiento ante el impacto del desempleo de las familias en situación de vulnerabilidad.

\section{Palabras Clave:}

Desempleo, afrontamiento, incertidumbre, inseguridad, metodología cualitativa. 


\section{Introducción}

Numerosos estudios demuestran que el empleo ocupa un lugar central en la vida de las personas (Anaf et al., 2013; Aristegui, Beloki y Mosteiro, 2012; Buendía, 2010; Jahoda, 1982; Weckström, 2012). Tener un empleo remunerado permite al individuo, además de satisfacer sus necesidades personales, integrarse en sociedad (Aristegui et al., 2012), mientras que para las familias el empleo constituye la primera fuente de seguridad material, social y psicológica (Buendía, 2010; Menaghan, 1991; Schliebner y Peregoy, 1994; Villardón et al., 2011).

Por tanto, el desempleo, y en especial el de larga duración, es un acontecimiento vital estresante que afecta no sólo a la persona desempleada, sino al conjunto del sistema familiar (Anaf et al., 2013; Buendía, 1990, 2010; Gough y Killewald, 2011; Janlert y Hammarström, 2009; Kalil y Wightman 2011; Menaghan, 1991; Neppl, et al., 2015; Schliebner y Peregoy, 1994; Villardón et al., 2011). En este sentido, las consecuencias para la persona desempleada van más allá de la mera pérdida de ingresos económicos, provocando profundos cambios en la estructura personal o familiar, que se manifiestan en tres dimensiones, psicológica, física y social, y el paso, en muchos casos, a la denominada clase de "nueva pobreza' (Dagdeviren, Donoghue y Meier, 2016)

Además, las familias con prole a su cargo resultan especialmente vulnerables al impacto del desempleo, puesto que, con la combinación de los estresores derivados de la situación de desempleo y las demandas de la parentalidad, los recursos emocionales de los individuos pueden verse menguados, lo cual puede reducir la capacidad de cuidar de los miembros de la familia (Schliebner y Peregoy, 1994; Weckström, 2012).

La literatura sobre afrontamiento y resiliencia nace en la década de los sesenta de disciplinas como la psicología y las ciencias de la salud, por lo que su foco de atención ha estado tradicionalmente puesto en el individuo -y posteriormente las familias- y en los atributos, habilidades, capacidades y actitudes que éste despliega a la hora de hacer frente a eventos estresantes o adversos (Lazarus, 1966, 1981, 1999; Lazarus y Folkman, 1984; Lazarus y Launier, 1978; Patterson, 2002a, 2002b; Werner y Smith, 1992). Pese a que se reconoce que el afrontamiento es un proceso dinámico que implica transacciones entre personas y sus entornos (Lazarus y Folkman, 1984), a esta perspectiva se le cuestiona, desde disciplinas como la sociología, la escasa o nula atención prestada a los factores estructurales más amplios (Folkman y Moskowitz, 2004). Ignorar elementos como el sexo/género, la clase social, la pertenencia a minorías, las prácticas culturales, el sistema redistributivo o la ideología enturbia la influencia de los factores estructurales en el estudio del afrontamiento. Esto implica retomar de nuevo el viejo - pero oportuno-debate sobre la agencia-estructura, también en la aproximación al afrontamiento y la resiliencia. En este sentido, investigaciones realizadas en los últimos años sobre el afrontamiento y la resiliencia ante la adversidad económica y el desempleo en contextos de crisis enfatizan la necesidad de incorporar esta perspectiva estructural, por su trascendencia/incidencia en los procesos de afrontamiento y resiliencia (Anaf et al., 2013; Dagdeviren, Donoghue y Meier, 2016; Dagdeviren, Donoghue y Promberger, 2016; Revilla, Martín y De Castro, 2017).

Contar con apoyo y protección social, entendido como el acceso a recursos materiales, emocionales e informacionales provenientes de las redes formales e informales, es, según la literatura, uno de los factores de protección más importantes en el afrontamiento y la resiliencia ante el desempleo y la vulnerabilidad social en contextos de (post)crisis económica, tal y como muestran distintas investigaciones llevadas a cabo en distintas comunidades autónomas (EDIS, 2010; Elorrieta, 2013; Comité Técnico de la Fundación Foessa, 2017) y en varios países tanto de la Unión Europea (Chzhen, 2016; Dagdeviren, Donoghue y Meier, 2016; Kroll y Lampert, 2011; Saccheto y Vianello, 2015; entre otros) como fuera de ella (Aytaç, Rankin e Ibikoglu, 2014; Israr et al., 2000; Martín Padilla et al., 2007; entre otros).

El apoyo o la protección pueden provenir de redes formales, es decir, de aquellos recursos institucionales regulados por políticas públicas en sus distintos niveles (estatal, autonómico, provincial y municipal) y de redes informales, como la familia extensa, las amistades, la comunidad, u organizaciones caritativas o del tercer sector (Dunst y Trivette, 1990). A su vez, el apoyo y la protección provistas o recibidas puede ser de carácter emocional, instrumental/material o informacional (Hegelson y Lopez, 2010). Finalmente, se distingue el apoyo activo, es decir, aquellas acciones o conductas específicas realizadas por terceras personas, las cuales se consideran como una expresión explícita de apoyo o asistencia del apoyo potencial, es decir, la percepción sobre la disponibilidad de apoyo en caso de necesidad existente que tiene una persona (Dunst y Trivette, 1990).

En las siguientes páginas, vamos a relatar cómo se percibe y se vive el impacto del desempleo de larga duración en distintos tipos de familias con prole de Bizkaia y en las estrategias de afrontamiento que utilizan para hacer frente a esta situación y a sus consecuencias, para después partir hacia la construcción de un modelo de afrontamiento ante el desempleo.

\section{Metodología}

Se ha optado por una metodología de carácter cualitativo (Creswell, 2007), por medio de entrevistas en profundidad individuales, para dar voz y profundizar en el significado subjetivo de las vivencias de muchas de las familias en situación de 
desempleo (Engel y Schutt, 2013). De este modo, y en consonancia con la metáfora del viajero, las personas entrevistadas han sido consideradas como participantes activas en la creación de significados, que contribuyen a la construcción de la experiencia de su mundo y, por ende, co-constructoras de conocimiento (Kvale, 1996; Kvale y Brinkmann, 2015).

Las entrevistas realizadas han sido semiestructuradas, ya que este tipo de entrevista otorga a ambos sujetos - entrevistador(a) y entrevistada/o- una amplia libertad, al mismo tiempo que permite asegurar que se tratan todos los temas relevantes y que se recoge la información necesaria. El guión que establece la persona entrevistadora dibuja las fronteras dentro de las cuales puede decidir no sólo el orden y la forma de formular las preguntas, sino también qué aspectos investigar en mayor profundidad (Ander-Egg, 2003; Corbetta, 2003; Ruiz Olabuénaga, 2012a, 2012b).

Se han llevado a cabo 23 entrevistas en profundidad a personas adultas -17 mujeres y 6 hombresprocedentes de familias biparentales (en las que uno o ambos progenitores están en desempleo), de familias monoparentales - la mayoría con progenitor mujer, razón por la que nos referimos a ellas como familias monomarentales-y de otras estructuras familiares ${ }^{1}$. Las personas seleccionadas han sido reclutadas, en primer lugar, por medio de organizaciones formales (Lanbide) y de organizaciones de carácter informal (banco de alimentos, asociaciones) de varios municipios de Bizkaia, y posteriormente, empleando la técnica de bola de nieve (Engel y Schutt, 2013). El trabajo de campo se ha desarrollado durante el primer semestre de 2016.
Los criterios de inclusión que definen la muestra de la población objeto de estudio han sido los siguientes: área geográfica, situación social, situación laboral, tipología familiar y procedencia (Cuadro 1).

\section{Resultados: un análisis descriptivo-interpretativo}

En este apartado, se presenta un análisis descriptivointerpretativo fruto de las entrevistas realizadas en el trabajo de campo, cuyo objetivo será transmitir fielmente el 'construct personal' (Ruiz Olabuénaga e Ispizua, 1989) de las personas participantes. Esta aportación descriptiva-interpretativa, basada fundamentalmente en análisis del primer ciclo de codificación (Miles, Huberman y Saldaña, 2014; Saldaña, 2016), es el paso imprescindible previo a la construcción de un modelo comprensivo de la realidad de estas familias. En este apartado, se desgranan por piezas - como si de un puzle se tratase- los relatos de las personas entrevistadas, piezas que, una vez unidas adecuadamente, permitirán visualizar la imagen que forman. Sólo comprendiendo las experiencias sobre el impacto del desempleo y su afrontamiento de las personas entrevistadas y sus familias, y los significados que atribuyen a dichas vivencias, podremos elaborar posteriormente un modelo de afrontamiento.

\subsection{El impacto del desempleo en los hogares: incertidumbre e inseguridad}

La pérdida del empleo supone una disminución de los ingresos en el hogar, lo cual produce un impacto económico que redunda en el conjunto del sistema

Cuadro 1. Criterios de inclusión

\begin{tabular}{|c|c|c|}
\hline Criterio & Criterio de inclusión & Definición/justificación \\
\hline Área geográfica & $\begin{array}{l}\text { Residentes en el territorio histórico de } \\
\text { Bizkaia. }\end{array}$ & $\begin{array}{l}\text { Particularidades del impacto del desempleo } \\
\text { en el contexto vizcaíno. }\end{array}$ \\
\hline Situación social & $\begin{array}{l}\text { Titulares de derecho a optar a prestaciones } \\
\text { contributivas y no contributivas (prestaciones } \\
\text { o subsidios por desempleo, RGI o AES) en los } \\
\text { últimos cinco años. }\end{array}$ & $\begin{array}{l}\text { Cumplir con los requisitos necesarios para } \\
\text { ser perceptoras de prestaciones contributivas } \\
\text { y no contributivas (prestaciones o subsidios } \\
\text { por desempleo, RGI o AES) en la actualidad o } \\
\text { haberlo sido en los últimos cinco años. }\end{array}$ \\
\hline Situación laboral & Desempleo de larga duración. & $\begin{array}{l}\text { Un año o más en el registro como } \\
\text { demandante de empleo y haber trabajado } \\
\text { previamente. }\end{array}$ \\
\hline Tipología familiar & $\begin{array}{l}\text { Familias biparentales o monoparentales con } \\
\text { prole a cargo }\end{array}$ & Afectación al sistema familiar. \\
\hline Procedencia & $\begin{array}{l}\text { Haber nacido en Euskadi o en comunidades } \\
\text { limítrofes. }\end{array}$ & $\begin{array}{l}\text { Sólo se incluye a la población autóctona, } \\
\text { dejando fuera a la población migrada de } \\
\text { otros países. Se pretende mostrar una nueva } \\
\text { realidad familiar. }\end{array}$ \\
\hline
\end{tabular}

RGI: renta de garantía de ingresos. AES: ayudas de emergencia social. Fuente: Elaboración propia. 
familiar (Anaf et al., 2013; Janlert y Hammarstrom, 2009; Kalil y Wightman, 2011; Neppl et al., 2015). Las familias entrevistadas han experimentado una pérdida en el poder adquisitivo y han visto afectada su capacidad de hacer frente a gastos, aunque no en la misma medida.

Para algunas familias, el hecho de perder un empleo ha supuesto una movilidad social descendente drástica, al pasar a formar parte de lo que Dagdeviren, Donogue y Meier (2016) denominan 'la nueva pobreza'. Este descenso supone un cataclismo para aquellas personas que, en un escenario de confianza propiciado por un empleo y la bonanza económica, formaron sus familias y adquirieron responsabilidades, gozando de cierto bienestar (Aristegui et al., 2017). Sin experiencia previa con la precariedad y la vulnerabilidad, de este grupo destacan dos aspectos relevantes: la pérdida repentina de estatus social -y cómo lo experimentan-y cómo afrontan sus nuevas circunstancias. El contraste en sus vidas antes y después del desempleo en un contexto de crisis económica les permite expresar sus nuevas circunstancias con un intenso sentido de conciencia sobre su movilidad al ‘submundo' de la vulnerabilidad y la pobreza:

Mira, por una amiga [...], ella nos decía: “¡Vete a la Cruz Roja, al banco de alimentos!”. ¿¿Qué era eso?! ¡Es que no sabíamos ni lo que era eso! 0 sea, no teníamos ni idea, ni lo que era un comedor social [...], ni lo que era el banco de alimentos, ni que la Cruz Roja te ayudaba... O sea, jes que vives como en otro mundo! Vives en el mundo de encima, ¿no? Y luego te bajas al submundo y dices, “¡Dios! ¿Qué es esto? ¡Si yo no me he enterado ni de que esto existía!” (M5MO).

Pese a la precariedad creciente y la reducción del gasto social (Dagdeviren, Donogue y Meier, 2016), otras familias no experimentan una movilidad social tan abrupta a raíz del desempleo, bien porque cuentan con una larga trayectoria en situación de vulnerabilidad o pobreza, bien porque aún cuentan con recursos propios (empleo de la pareja, ahorros, patrimonio) que las protegen del impacto.

En este sentido, itinerarios intermitentes de empleodesempleo, así como recorridos continuados en empleos poco remunerados, conllevan que, para algunas familias, la vulnerabilidad o la pobreza hayan sido una constante en sus trayectorias de vida. Consecuentemente, algunas familias llegan a normalizar la experiencia e incluso la interiorizan como parte de su propia identidad (Aristegui et al., 2017; Dagdeviren Donogue y Meier, 2016). Esta ‘vieja pobreza' (Dagdeviren, Donogue y Meier, 2016), arraigada en las formas de vida de las personas, se refleja en una desesperanza continua con respecto a una mejora de sus condiciones futuras, coincidiendo con Aristegui et al. (2017):

Yo vivo al día, pero desde hace mil años, o sea, siempre he vivido al día, nunca he tenido ahí algo que te sobre o... Con llegar al fin de mes, ya me conformo, que llegue el día..., vas a comer mañana y tengas $10 €$ en la cartera, con eso, vamos, ya, me conformo ( $\left.\mathrm{M}_{3} \mathrm{MO}\right)$.

Los discursos de las familias que aún cuentan con recursos propios que las protegen giran en torno a la capacidad de agencia para hacer frente a la precariedad y la vulnerabilidad, haciendo énfasis en una forma de vida basada en la austeridad y en la previsión como elementos fundamentales para capear el impacto del desempleo en un contexto de crisis económica:

Pues yo creo que eso, que al tener..., al no haber vivido nunca... al límite de las posibilidades, pues ahora [con] la crisis... más o menos vivimos igual. 0 sea, económicamente no nos..., no nos hemos sentido..., no nos..., no estamos sufriendo, o sea, porque siempre hemos estado con lo..., con lo justo, aunque tuviésemos más, vivíamos con lo justo, entonces eso lo teníamos ahorrado y entonces la crisis no... 0 sea, a pesar de quedarme yo en paro, tenemos menos dinero, pero seguimos viviendo más o menos igual. Que no hemos tenido grandes..., o sea, que no hemos tenido que renunciar a cosas (M2OBI).

Sí, yo creo que la cosa sigue igual, porque quizás hemos tenido la suerte de que no somos muy suntuosos, no... no entendemos la calidad de vivir que como de gastar, no somos gastadores, nos gusta..., pues eso, disfrutar, pero no..., no hemos tenido gastos, yo especialmente, yo bueno, soy especialmente austero, pero bueno $\left(\mathrm{H}_{13} \mathrm{BI}\right)$.

En cualquier caso, existe una sensación de inseguridad y de incertidumbre inherente a la coyuntura económica. Incluso familias donde el impacto del desempleo ha sido menos perjudicial manifiestan una conciencia de la fragilidad de las condiciones materiales, cuyo cambio a peor podría comprometer su capacidad de agencia:

Que muchas veces, cuando tienes mucho, mucho..., no quiere decir que sea duradero. Te quiero decir... Ya te digo, vivir al día es lo mejor (M18BI).

Yo creo que más el miedo a hacer cosas..., el... “por si acaso", el "ahora estamos bien”, pero si a él le dicen que no tiene trabajo, entonces ya dejamos de estar bien. Entonces yo pienso más el miedo, que... lo..., porque yo sé que hay gen..., hay gente que sí lo está pasando mal; nosotros..., no. Pero tenemos miedo, a que..., a que nos llegue (M2OBI).

Entonces, ahora dependemos del trabajo de él, entonces eso es lo... lo importante. Y como tampoco tiene un trabajo...., o sea, está indefinido, pero bueno, lo de indefinido hoy en día es tal, pues como vivimos en siempre con miedo, pues eso es lo que nos para a hacer cosas. 
O sea, que inclu... hacemos menos cosas que las que podríamos, por miedo (M2oBI).

A ve... a ver si me entiendes..., cuando hemos estado bien, porque había dinero para todo, no hacía falta hacer estas cosas, igual hacía falta, pero no teníamos como la consciencia de que hacía falta, ¿no? Era como, "bueno, de los pobres se encarga Cáritas, de los pobres se encarga la Cruz Roja... ". Que no, que mañana puedes ser tú, que los pobres nos pertenecen a todos, que todos tenemos que ayudar (M5MO).

\subsection{Estrategias de afrontamiento}

Las personas emplean estrategias de afrontamiento de distinta naturaleza de cara a hacer frente a diversas situaciones. Siguiendo la clasificación de estrategias de afrontamiento clásica propuesta por Lazarus y Folkman (1984), únicamente se presentarán en este apartado aquellas estrategias orientadas a la resolución de problemas o estrategias de carácter práctico que emplean las familias de cara a afrontar el desempleo y su consecuente pérdida de poder adquisitivo. Esto implica que se dejarán de lado las estrategias orientadas al manejo de emociones. Así pues, entre las estrategias empleadas por las familias para hacer frente al desempleo y a la vulnerabilidad, se distinguen aquellas orientadas a la reducción del coste vida y aquellas dirigidas a la búsqueda de nuevas fuentes de provisión.

\subsubsection{Estrategias orientadas a la reducción del coste vida}

De cara a reducir el coste de vida, aquellos gastos considerados más superfluos, menos esenciales, son los primeros en verse afectados, bien reduciéndolos o bien suprimiéndolos, al igual que lo observado en la literatura (Aytaç, Rankin e Ibikoglu, 2014; Chzhen, 2016; Saccheto y Vianello, 2015). A medida que la situación de adversidad económica se prolonga en el tiempo, la balanza de lo prioritario se va inclinando a favor de lo superfluo, al mover cada vez más elementos previamente considerados como necesidades al plato de lo 'no tan necesario' o 'menos necesario'. Al final, las necesidades se reducen a las más básicas:

Le decimos "estamos solos, con que no te falte de comer, que es lo principal para que no te mueras, pues... lo otro es... un lujo, ¿no?”. Creo yo... (M4MO).

En este sentido, tal y como reflejan los datos del DeustoBarómetro Social (2013-2017), el gasto en actividades de ocio y tiempo libre es el que más se ha visto afectado, por lo que, entre las estrategias utilizadas para reducir el coste de vida, han de mencionarse las utilizadas en relación a aquellas actividades que requieren cierto gasto - por mínimo que sea-, cuya frecuencia se reduce o que incluso se deja de hacer:
Ahora mismo, no, bueno, llevamos una temporadita que no podemos ir, vamos, jni al cine! ¿Ir al cine? Vamos, ahora mismo es un lujo para nosotros $\left(\mathrm{H}_{1} \mathrm{BI}\right)$.

En relación con las oportunidades formativas, las familias reducen o suprimen las actividades extraescolares de sus hijos e hijas. En el caso de quienes llevan una trayectoria de vulnerabilidad e incertidumbre constantes, su prole no puede optar a realizar ninguna actividad extraescolar que suponga un coste económico. En el caso de las familias que vivieron en una situación de bonanza previa, puede implicar que no puedan ofrecer las mismas oportunidades a toda su prole:

Otras amigas que han ido en verano a esos cursos de inglés al extranjero, que su hermana mayor sí fue y ya la segunda no ha podido ir, porque le hemos dicho [que] no estamos como para pagar un curso de ésos, pero vamos, tampoco son cosas que tiene que hacer todo el mundo $(\mathrm{HgBl})$.

Entre las estrategias utilizadas relacionadas con la vivienda, cabe destacar la reducción del consumo de suministros como el agua, la electricidad o el gas. Asimismo, también emplean estrategias relativas al lugar de residencia, de forma que las familias, bien se mudan a viviendas más asequibles, vuelven al domicilio familiar de origen, posponen compras de viviendas o bien migran desde otras comunidades autónomas o se plantean migrar a otros países. Estas estrategias son también las adoptadas en otros países por personas en situaciones similares (Aytaç, Rankin e Ibikoglu, 2014).

Añadido a ello, adoptan estrategias relacionadas con los hábitos de consumo. Uno de los mecanismos empleados consiste en comprar alimentos de marca blanca, comprar la ropa y el calzado en periodo de rebajas o en establecimientos más asequibles (Primark, AliExpress, mercadillos). Como forma de disminuir gastos, también realizan la compra recorriendo los supermercados locales en busca de las ofertas disponibles en cada uno de ellos y compran los productos ofertados en grandes cantidades para almacenarlos para el futuro próximo. Destacan además el reciclaje, y el uso de ropa y otros objetos (cunas, juguetes) de segunda mano.

También se relacionan con los hábitos de consumo los cambios en la alimentación diaria: la reducción del consumo de carne (en especial, la roja) y pescado, el aumento del consumo de alimentos con altos contenidos de carbohidratos (arroz, pasta, patatas), la disminución de la cantidad de comida e incluso, en algunos casos, la reducción del número de comidas diarias. Sin embargo, estos cambios en la alimentación son estrategias que utilizan las personas adultas para que su prole pueda seguir manteniendo, en la medida de lo posible, una alimentación suficiente, sana y variada, pese a que ello implique sacrificar la propia: 
Pues el lunes, el martes y el miércoles, como macarrones. Y no me importa. A mí no me importa en absoluto. Luego ya, para la noche, pues para la cría, pues igual le cojo unas anchoítas, un poco de... del más barato, no le voy a sacar, claro, no voy a comprar un pescado de esos caros, pero cojo unos poquitos para ella y tal, y así... [...]. Que yo lo único que pienso es en la cría. Porque yo, muchas noches, igual patatas fritas o una pieza de fruta o una lata o lo que sea, pero la cría, sus comidas. Y menos mal que come en el colegio, pero cenar, cena en casa $\left(\mathrm{H}_{2} \mathrm{MO}\right)$.

Finalmente, las familias con menos recursos económicos enfatizan la creatividad y el ingenio para reducir costes de la vida cotidiana:

-M6M0: Y luego es otra de las cosas, ¿cómo haces que una misma comida sea diferente en seis ocasiones?

$-\mathrm{I}$ ¿ ¿El arroz, por ejemplo?

-M6MO: Eso es, es como... “¿otra vez?”. Claro, tú eres mayor, y dices "bueno", aunque estés un poco cansada..., pero es lo que hay; pero... una niña no te come siempre lo mismo. Es como los nuggets. A mí los nuggets me resultan muy caros, entonces los hago yo... Estiro con una pechuga, ¿eh? -esto es sólo con una pechuga-, yo estiro mucho, mucho, mucho la pechuga y hago formas con lo que tiene mi hija de plastilina, lo tengo al lado y voy pegando, y hago formas, y luego lo rebozo, tres veces, para hacerlo más gordo [ríe].

\subsubsection{Estrategias dirigidas a la búsqueda de nuevas formas de provisión: movilización de recursos propios y búsqueda de apoyo social}

Además de reducir el coste de vida, las familias emplean estrategias dirigidas a la búsqueda de nuevas formas de provisión (Saccheto y Vianello, 2015), bien movilizando recursos propios del hogar y de sus miembros, bien buscando y movilizando recursos fuera del ámbito familiar nuclear que pueda ofrecer apoyo y protección.

Cuando las personas pierden sus empleos, las familias emplean sus propios ahorros o patrimonio, en caso de tenerlos, como estrategia para compensar la pérdida de poder adquisitivo. Sin embargo, esta estrategia tiene una limitación temporal, y a medida que estos recursos se van agotando y la situación de desempleo se sostiene, las familias se ven forzadas a realizar otro tipo de acciones. Entre las prácticas adoptadas por las familias que previamente se encontraban en una situación de cierta comodidad, cabe destacar aquellas provenientes del mercado, como hacer uso de préstamos bancarios, créditos - lo que implica un aumento de su deuda- o el pago a plazos, tal y como muestran también los resultados reportados por Chzhen (2016) en su estudio. Por otro lado, muchas familias aceptan empleos en condiciones precarias, especialmente en la denominada economía sumergida, cuyos ingresos añadidos les permiten cierto margen de maniobra en el afrontamiento de sus gastos. También se adoptan estrategias orientadas a mejorar las posibilidades de encontrar un empleo, ya sea realizando cursos de formación o incluyendo a otros miembros de la unidad familiar (prole y cónyuge) en la búsqueda de empleo, prácticas que implican cambios en la organización familiar (Aytaç, Rankin e Ibikoglu, 2014). Al mismo tiempo, las familias adoptan otros mecanismos, como la venta y empeño de objetos personales, como forma de obtener ingresos extras.

En definitiva, una vez agotadas las vías de aprovisionamiento habituales y los recursos disponibles entre los miembros, las familias se ven obligadas a "pues eso..., pffff..., sacar recursos de donde no los hay" (M23BI).

Por otra parte, entre las estrategias de búsqueda y movilización de recursos fuera del hogar, destaca la búsqueda de apoyo social en los distintos sistemas de protección como estrategia de afrontamiento esencial empleada por las familias vizcaínas, hecho que coincide con investigaciones realizadas en otros países (Chzhen, 2016; Martín Padilla et al., 2007), así como en Euskadi y en España (Buendía, 2010; Comité Técnico de la Fundación Foessa, 2017; EDIS, 2010; Lorenzo, 2014; Martínez Virto, 2017). Se acude tanto a redes formales e institucionales como a redes informales, entre las que destacan la familia extensa, las amistades y la comunidad.

En un primer momento, las familias movilizan recursos de las redes formales; es decir, aquellos recursos institucionales regulados por políticas públicas en sus distintos niveles (estatal, autonómico, provincial y municipal). De estos recursos, destacan la prestación por desempleo, el subsidio y los programas para la promoción de empleo para las personas desempleadas, y las becas para los niños y niñas en edad escolar (matrícula, comedor, material escolar). Una vez agotadas las prestaciones por desempleo y los recursos propios, las familias vascas cuentan con otra serie de prestaciones a la hora de hacer frente a gastos básicos. La renta de garantía de ingresos (RGI), en sus diversas modalidades (bien como complemento de ingresos provenientes de un empleo, bien en su modalidad básica, bien con el complemento de vivienda), junto con las ayudas de emergencia social (AES), actúan de malla de seguridad material vital, permitiendo no sólo hacer frente a los gastos básicos habituales, sino, en el caso de las AES, también a aquellos gastos extraordinarios que surgen de forma inesperada (aunque de forma puntual). Sin embargo, pese a que la percepción de prestaciones, sean contributivas o no contributivas, otorgue cierta seguridad, al proporcionar a las familias ingresos de forma periódica y estable, éstas resultan insuficientes para afrontar todos los gastos, por lo que las familias se ven obligadas a buscar formas de provisión complementarias de forma habitual:

- M4M0: Pues que no te llega igual, con $800 €$ tienes que pagar la luz, el agua, el piso, comer, vestir. Pues todo no llega con $800 €$. 
[...]

-I: ¿Cómo te las apañas?

-M4MO: Pues lo que me ayuda mi ama [madre]; los fines de semana viene aquí, me suele echar una mano. Hoy vendrá a dormir, luego se va el domingo... Y eso, y lo que vendo por internet, ya te digo.

Sin embargo, no todas las prestaciones tienen el mismo significado para quienes las perciben y, por tanto, condicionan el concepto que estas personas tienen de sí mismas. Las prestaciones contributivas, vinculadas al empleo y, por tanto, asociadas, a la identidad de trabajador(a), se conciben de forma simbólica como diametralmente opuestas a la percepción de prestaciones como la RGI:

Porque eso..., cuando me dan el paro es porque yo, antes..., o sea, digamos que es... cuando te retienen cosas; o sea, cuando te retienen dinero, o sea, de tu trabajo..., pues tú cobras en bruto tanto, ¿no? Y te llevas tanto. Pues una parte es precisamente para el paro. Si no, no te la darían, es tuya, de por... Porque yo tampoco estoy a gusto, cobrando el [sic] RGI, porque yo siempre he sido una traba..., una 'curranta' nata, yo es que esto de... de vivir así... tampoco es lo mío (M14MO).

La suspensión de este tipo de prestaciones, por tanto, es uno de los principales miedos que comparten las personas perceptoras, como bien se ha comentado, ya que pone en jaque la subsistencia del hogar, desestabilizando el sistema familiar. Cuando se da una ausencia total de ingresos formales, es cuando el apoyo proveniente de las redes informales resulta clave:

-M17CC: [La RGI] me la cortaron dos meses. La (arrendadora) del otro piso no aguantó, le explique la situación, le dije que me tenía que hacer un contrato nuevo en el que estaría yo sola, y me dijo que yo sola que no. Entonces claro, me lo cortaron y le dije yo "pues no te la puedo pagar, pues te quedas con el piso y te quedas con todo". -I: ¿Cómo lo hiciste durante esos dos meses que, de repente, te suspenden la RGI?

- M17CC: Como me vine a casa de mis padres, no tuve problemas; una vez que me empadroné en casa de mis padres, me la volvieron a conceder, entonces ya está.

Las redes familiares constituyen la fuente de apoyo informal más importante de cara a hacer frente al desempleo para las personas entrevistadas y sus familias, en consonancia con lo que se recoge en la literatura (Lorenzo, 2014; Requena, 2011; Vila, 2014). La familia extensa, especialmente las personas mayores con sus pensiones, proporciona apoyo material indispensable a sus miembros más vulnerables o en situación de necesidad. Así, contribuyen al cuidado de la prole y ayudan con los gastos básicos (vivienda, energía, ropa, comida), constituyéndose en pilar fundamental para poder hacer frente a los gastos habituales y a las contingencias (gastos inesperados o extraordinarios), al actuar como protectores de la economía familiar. Este tipo de apoyo es también un importante soporte emocional y constituye una fuente de seguridad ante la incertidumbre:

Y sé que, si un día no tengo para hacer la compra, me viene mi madre y mi madre me hace la compra. Yo ahí tengo una suerte inmensa, o sea, tengo una suerte inmensa (M17CC).

Sin embargo, no todas las personas disponen de una familia extensa, y tenerla tampoco garantiza siempre la disponibilidad de un factor de protección ante la adversidad; a veces, tenerla y no poder acudir a ella puede ser un factor de riesgo. Un ejemplo de percepción de falta de apoyo es el siguiente comentario:

Porque es..., nos tenemos sólo a nosotros. Sí, no, no tenemos apoyo de ninguna de las dos familias..., sólo no... nos tenemos a nosotros (cuatro). A mi madre, alguna vez le he pedido que me dejaría dinero, ni 50, ni 100, 20 euros; me ha dicho que me vaya al pres... a, al banco a pedir un préstamo. Entonces, antes que pasar por esa situación, no, prefiero no pedir nada ( $\left.\mathrm{H}_{19} \mathrm{BI}\right)$.

Otras familias no tienen la capacidad para apoyar y proteger a sus miembros, bien porque los mecanismos de protección se han visto estresados a raíz de la crisis económica, o bien porque también forman parte de colectivos que tradicionalmente han sido objeto de protección:

Pero bueno, no, yo..., mis padres están jubila..., son pensionistas y... no, no les suelo pedir, bastante que no..., que me pagan, me pagan la comunidad y eso, me pagan ellos, gastos de la casa (M15MO).

La red de amistades se constituye como otra fuente de apoyo importante para las familias en situación de desempleo. A diferencia de la red familiar, no todas las personas conservan su círculo de amistades tras perder su empleo. En este sentido, mencionan que en estas situaciones de vulnerabilidad y adversidad es cuando se prueba la amistad de las personas:

Porque lo que te das cuenta es quién está y quién no está, o quién decía estar y no ha estado nunca, que normalmente es lo que pasa..., que normalmente... te quedas más sola que la mojama. Pero bueno, te sirve para hacer limpieza (M21M0).

Al igual que la familia, las amistades proporcionan apoyo material y emocional. Este apoyo por parte del grupo de iguales conserva el sentido de pertenencia al grupo:

Gracias a Dios, tenemos buenos amigos y estamos... muchas veces son ellos "venga, vamos 
a cenar, que os invito yo". Entonces, tipos de amistades, ahí no ha cambiado nada, siguen siendo los mismos. Entonces eso de "como tienes dinero, vamos y estáis con nosotros, y ahora que no tenéis, os dejo" no existe en nuestro círculo de amistades, estamos todos bien compenetrados, son amigos de toda la vida y nos llevamos bien (M10BI).

La integración en redes comunitarias facilita una mayor diversidad de estrategias resilientes que pueden contribuir a la recuperación y supervivencia de los hogares (Revilla, Martín y De Castro, 2017). En este sentido, podemos afirmar que las familias obtienen apoyo por parte de su vecindario, de redes de solidad informales, de diversas organizaciones caritativas y del tercer sector, así como de comunidades religiosas.

Las personas entrevistadas, además de acudir a redes familiares y amistades, también acuden a diversas organizaciones caritativas (banco de alimentos, Cáritas, Cruz Roja) cuando la ayuda proveniente del sector público resulta escasa para hacer frente a la adversidad:

Yo vivo de la caridad, directamente. Y es que no me da vergüenza decirlo, porque es lo que hay en este país en este momento. Aquí estamos viviendo de la caridad millones de personas. Y nosotros somos unos de ellos, que vivimos de la caridad. De que me ayude mi madre un poquito, mis suegros nos ayudan también, mi cuñado también nos echa una pequeña mano, luego lo que cobramos de Lanbide y estas mujeres de aquí [en referencia a las voluntarias de una asociación del municipio], que, por suerte, nos dan un paquete de comida bastante majo, que al menos podemos comer al mes $\left(\mathrm{H}_{1} \mathrm{BI}\right)$.

Además del entramado asociativo y del vecinal, las personas acuden a comunidades virtuales de solidaridad. Estos espacios de solidaridad interpersonales, frecuentados en su mayoría por mujeres, se organizan a través de grupos en distintas plataformas (redes sociales, páginas web) para donar e intercambiar aquello que ya no les sirve. Esta ayuda mutua se configura como espacio de solidaridad ejercida de forma horizontal, donde lo intercambiado trasciende lo material y se generan lazos:

Lo que pasa que se te agudiza el ingenio, igual que a ti, se le agudiza a todo el mundo; entonces, se han creado tropecientos grupos de... Yo, por ejemplo, estoy en uno de Facebook [...]. Que por eso agencias ropa nueva, claro, entonces en ese grupo estamos muchísima gente así. Entonces lo que va... lo que a uno le va quedando pequeño. pero que está... útil, se lo va pasando al siguiente... entonces yo llevo dos años así, porque... porque no, es que, si no, no. [...] Y al, al final, claro, si... normalmente, si... si andas mucho, pues normalmente te conoces entre... entre las que somos del grupo, entonces al final, pues sí, como se hacen quedadas y vas y..., pues bueno, se crea un buen rollito, que por lo menos, un buen rollito que no cuesta dinero..., por lo menos, durante media hora te ríes, estás a gusto, estás con gente que está pasando por lo mismo que tú, entonces, pues bueno, no te ves tan desplazada (M21M0).

Las comunidades religiosas también constituyen en importante fuente de apoyo emocional y espiritual para las personas:

Sí, sí... Pues cómo hablan, y que siempre tienen fe, pues gente positiva, ¿no?, no gente que te dice, "pues, con tres hijos, no vas a salir o...", [cosas] así, que hunden, igual, psicológicamente. Aquí no, aquí siempre están, que siempre se ayuda para todo (M4MO).

Pese a que el apoyo social proveniente de las diversas redes resulta fundamental para muchas familias, no está exento de costes emocionales y sociales para sus miembros. Tener que depender de recursos que no sean propios para la subsistencia del hogar genera sentimientos de vergüenza y de culpa, y puede suponer un estrés añadido a la propia situación de adversidad. Admitir la necesidad de ser ayudado significa hacer visible esta vulnerabilidad, minando la propia autoestima:
Soy muy, muy orgullosa. A mí, toda la vida me han enseñado a ayudar y a dar, pero no me han enseñado a pedir y recibir. Y yo lo paso muy mal... Yo, cuando alguien me quiere ayudar igual, pues eso, cuando le tengo que pedir dinero, a ver, ¡lo necesito!, es ya de "pfff, o sea, me han embargado la cuenta, no tengo dinero", no es "te pido porque tengo, para un capricho o para equis"; es "no tengo, tengo cero y tengo que pagar la factura. Tengo cero". Y entonces la pido, pero si no, no la pido. Y si me la ofrecen, pfff..., me cuesta mucho decir que sí, aunque sepa que la necesito... Me cuesta mogollón aceptar que es verdad, que lo necesito, no... no sé ( $\left.\mathrm{M}_{5} \mathrm{MO}\right)$.

En este contexto, la reciprocidad en la relación emerge como aspecto a tener en cuenta en el efecto que tiene el apoyo informal en las personas, es decir, si la relación de ayuda es percibida como equilibrada o no en cuanto al rol de provisión y recepción adoptado. Cuando se siente que la ayuda es mutua, que la relación está compensada, estos costes emocionales y sociales se contrarrestan y, por ende, el apoyo es percibido como más beneficioso:

Económicamente, pues igual mi familia me dice “oye, que tengo que pintar el pasillo", aunque lo tengan pintado hace un año, "saca presupuesto y me lo pintas" [...], o "me voy de vacaciones, cuídame la casa" y cuando vienen "te he traído esto" o "te doy tanto por cuidarme la casa". Y vamos, por una parte, saben cómo estamos y en ciertas pequeñas cosas sí te ayudan, no son todos los días, sí de vez en cuando, porque 
tampoco quieren que yo me sienta siempre como si fuéramos ahí los marginados, pero no, yo creo que la familia hace mucho en mi casa, más que otra cosa con el ánimo ( $\left.\mathrm{H}_{10 \mathrm{BI}}\right)$.

Finalmente, entre las estrategias de búsqueda de fuentes alternativas de provisión y cuando los demás mecanismos y redes de protección fallan, están aquellas prácticas “de supervivencia irregulares” o “de exclusión” (Martínez Virto, 2017). Este tipo de estrategias únicamente han sido referidas de forma residual por una de las participantes, quien, debido a su falta de ingresos en el último año y medio (por suspensión de la RGI), afronta sus gastos mediante la venta de objetos robados y drogas:

-M6M0: La verdad [es] que, con la marihuana, me voy sacando unos $300 €$ al mes.

$-\mathrm{I}:$ ¿Al mes?

-M6M0: Sí, y no vendo mucho, o sea, vendo poquitos a poquitos, si necesito algo más, pues... vendo un poquito más. Luego vendo cosas, tengo un amigo que es..., trabaj..., él roba y yo le vendo la mercancía..., entonces me saco un tanto por ciento. Ahí es como, tengo esos, cuando me vienen esos imprevistos es lo que tengo que hacer; llamar a mi amigo para que me deje... y así poder pagar, eso es así.

\section{Hacia la construcción de un modelo de afrontamiento}

Partiendo de la fase descriptiva-interpretativa previa, y tomando como base el segundo ciclo de codificación (Miles, Huberman y Saldaña, 2014; Saldaña, 2016), se ha procedido a la construcción de un modelo explicativo-interpretativo comprensivo del afrontamiento ante el impacto del desempleo en las familias con prole a su cargo. Para ello, se han tomado como ejes vertebradores la categoría de acceso a recursos económicos, por un lado, y la de disponibilidad de apoyo social, por otro, por su influencia en la valoración que realizan las familias de su propia situación, sus expectativas y aspiraciones futuras, y en las estrategias de afrontamiento empleadas.

\subsection{Acceso a recursos económicos}

El acceso a recursos económicos emerge como categoría determinante en la evaluación que realizan las familias sobre su situación actual y sus perspectivas de futuro. Por tanto, en función de la naturaleza de estos ingresos (fuente, estabilidad/ inestabilidad, suficientes/insuficientes), las estrategias empleadas variarán. Se distinguen tres grandes grupos: 1) familias que sobreviven día a día; 2) familias con dificultades para llegar a fin de mes; $y$ 3) familias cuyo futuro no está asegurado (Figura 1).

\subsection{1. "(Sobre)viviendo día a día": rozando la exclusión social/en el precipicio de la exclusión social}

Estas familias carecen de ingresos formales (debido a que han agotado todas las prestaciones, se les ha suspendido o no cumplen requisitos para acceder a ellas), o estos ingresos son de carácter muy inestable y escaso. La incertidumbre impregna no sólo el futuro próximo, sino también el inmediato; la subsistencia de los miembros de la familia es una cuestión que se va resolviendo - o no- día a día. Esto se traduce en un miedo constante a no poder satisfacer las necesidades más básicas de su prole, a perder su casa y ser desahuciadas, a que

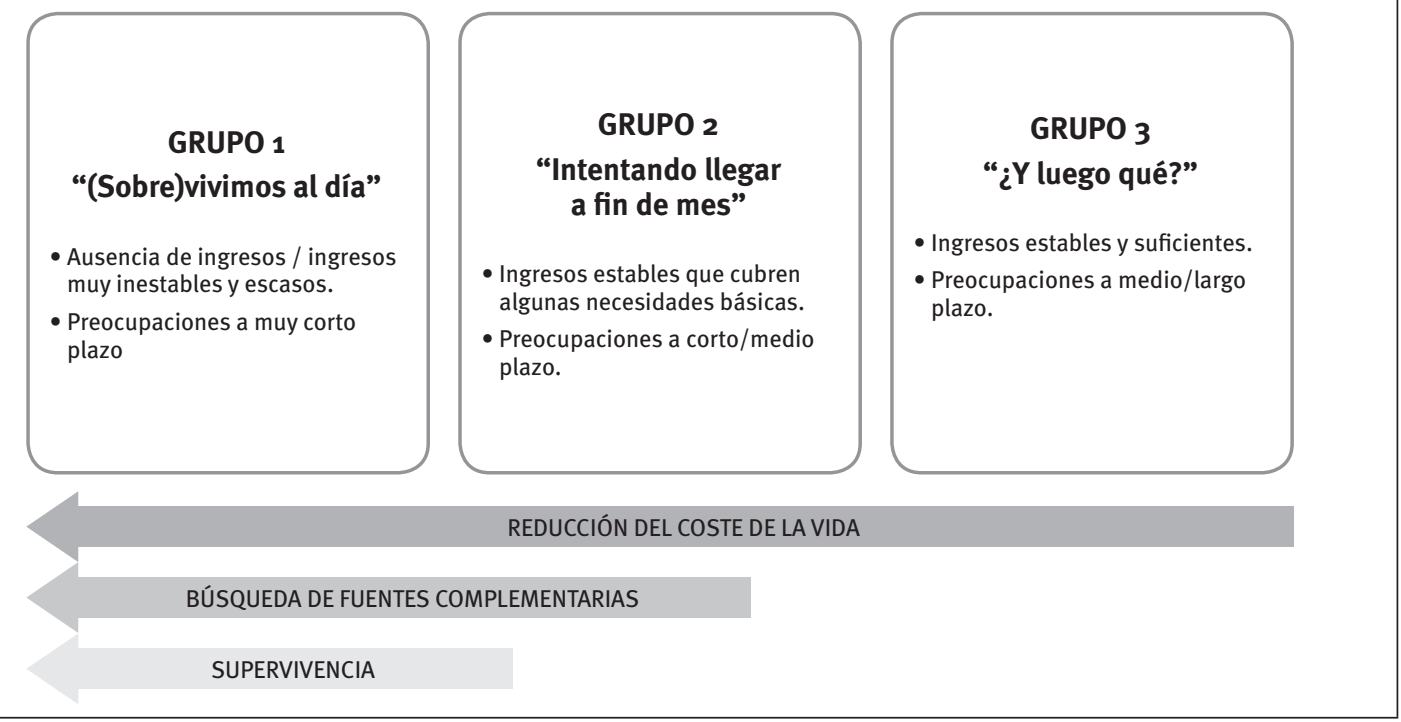

Fuente: Elaboración propia. 
les corten los suministros (electricidad, gas, agua), y una incapacidad absoluta de planificar a futuro. Aspiran a volver al mercado laboral, aunque sus expectativas no son nada halagüeñas. Consideran que la mejora de sus condiciones no depende tanto de su capacidad de agencia, sino de un profundo cambio estructural. De cara a afrontar esta situación, hacen uso de todos los recursos a su alcance: reducen al máximo los costes de vida y tratan de buscar formas alternativas de ingresos, aunque esto implique -en algunas ocasiones- adoptar estrategias más drásticas (venta de drogas, robos) si de ello depende su supervivencia y, especialmente, la de su prole. Las familias en esta situación son las más cercanas a la exclusión social. En este sentido, sólo la disponibilidad de apoyo social proveniente de una red -tanto formal como informal- sólida y con capacidad de protección podrá contrarrestar esta inseguridad y disminuir su vulnerabilidad a la exclusión social:

Más o menos, pues vengo eso, vivir día a día. O sea, yo no te puedo decir “¿cómo has sacado el mes?”, no tengo ni idea, no sé siquiera cómo voy a pagar mañana, o sea, para que te hagas una idea, yo hoy tengo que coger leche, entonces, la manera que he tenido de sacar dinero es: ayer, trabajé un par de horas cuidando a una cría, y gracias a eso, tengo dinero. Entonces hoy puedo comprar leche, mañana no lo sé, pero hoy por lo menos, hoy sí. Y más o menos pues eso, al día (M6MO).

\subsection{2. "Haciendo malabares para llegar a fin de mes / "La cuestión está en llegar a fin de mes": entre la exclusión y la inclusión}

Estas familias cuentan con ingresos estables con los que afrontan los gastos derivados de sus necesidades más básicas mes a mes (vivienda, energía, agua). La naturaleza periódica y estable de estos ingresos, en su mayoría provenientes de varios tipos de prestaciones (en sus distintas modalidades), les otorga la capacidad de asumir gastos fijos básicos, proporcionando cierta sensación de seguridad. Tienen cierta capacidad de planificación a futuro, aunque ésta se limita a un futuro inmediato; organizan la economía doméstica priorizando los gastos mensuales fijos, por lo que todo gasto extraordinario o inesperado resulta altamente desestabilizante. Se distinguen aquellas familias con una trayectoria continuada de vulnerabilidad, quienes viven en una "eterna incertidumbre" (Aristegui et al., 2017), de aquellas que han experimentado una movilidad social descendente más abrupta y son parte de la nueva pobreza (Dagdeviren, Donoghue y Meier, 2016). Las primeras son más escépticas respecto a la mejora de su vida futura, y especialmente con relación a su reincorporación al mercado laboral, mientras que las segundas aún aspiran a ello. Sin embargo, ambas comparten el hecho de que, si bien pensaban que esta situación de vulnerabilidad estaría limitada en el tiempo, se está alargando más de lo previsto y ven limitada su capacidad de agencia. Entre sus preocupaciones principales, está el futuro de su prole, es decir, que tengan las oportunidades necesarias para vivir mejor. Las estrategias de afrontamiento que utilizan estas familias son, principalmente, un fuerte reajuste de la economía doméstica con reducción de gastos y la búsqueda de fuentes complementarias de ingresos. Esta necesidad de buscar ingresos extras (economía sumergida, apoyo económico familiar) y fuentes de provisión alternativas (bancos de alimentos), que al principio se concebía como algo puntual y propio de la fase de ajuste, se convierte en una constante: "ya se ha convertido en un goteo [...], pero el goteo es constante" ( $\left.\mathrm{H}_{11} \mathrm{BI}\right)$. De nuevo aquí, la disponibilidad de apoyo social proveniente de una red sólida y con capacidad de protección es clave para hacer frente a la vulnerabilidad derivada del desempleo mes a mes, así como a las contingencias que puedan surgir.

\subsection{3. “¿Y luego quée?": manteniéndose en la inclusión}

Estas familias cuentan con ingresos estables provenientes del empleo bien remunerado de algún miembro de la familia, generalmente del progenitor o progenitora. El impacto del desempleo se manifiesta, sobre todo, en el plano psicológico, y afecta a los dinamismos vitales (autoconcepto, autoestima, identidad), puesto que para estas familias no implica una situación de privación material o de adversidad económica. Cuentan con ingresos estables y suficientes que les dan independencia y seguridad, por lo que carecen de la necesidad de buscar fuentes complementarias. Dado que su capacidad para hacer frente a la satisfacción de necesidades básicas no peligra, ni en el presente ni en el futuro próximo, sus preocupaciones se sitúan a medio/largo plazo y giran en torno a cuestiones como la pensión de jubilación o las condiciones de los empleos a los que podrán optar en el futuro, o sobre las oportunidades futuras para su prole. Las estrategias utilizadas por estas familias son principalmente las relacionadas con la reducción del coste de vida. A diferencia de los otros dos grupos, estos mecanismos de afrontamiento se limitan a reducir o eliminar aquellos gastos más superficiales, sin que ello implique comprometer las necesidades básicas. Siendo conscientes de la fragilidad de las condiciones materiales, también expresan el miedo a que éstas cambien, y por ello, emplean estrategias preventivas (por ejemplo, posponer la compra de una vivienda). Por tanto, para muchas de estas familias el desempleo de uno de sus miembros no implica cambios significativos en su estilo de vida, aunque no tengan la certeza absoluta de que su situación vaya a mantenerse en el tiempo, ni a medio ni a corto plazo.

\subsection{Disponibilidad de protección y apoyo social}

La disponibilidad de protección y apoyo social emerge como categoría determinante en la 

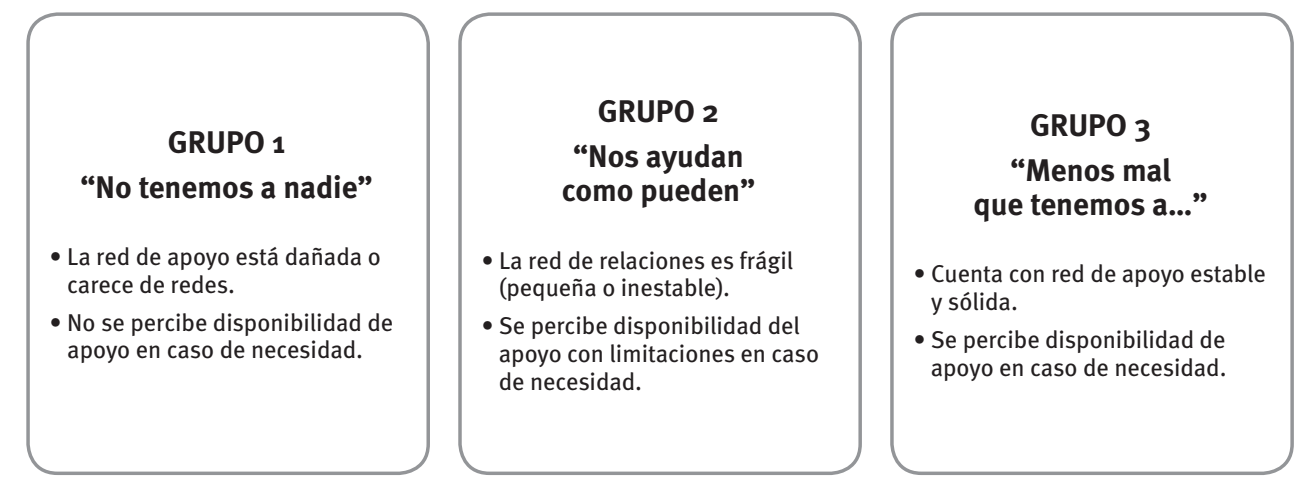

RECURSOS PROPIOS

RED FORMAL E INFORMAL

RED FORMAL E INFORMAL

Fuente: Elaboración propia.

evaluación que realizan las familias sobre su situación actual y sus perspectivas de futuro. Por tanto, en función de la naturaleza la red de apoyo potencial (tamaño, solidez/fragilidad, capacidad/ incapacidad), las estrategias empleadas de movilización de recursos variarán. Se distinguen tres grandes grupos: 1) familias que carecen de apoyo potencial; 2) familias con apoyo potencial limitado; y 3) familias que cuentan con apoyo en caso de necesidad (Figura 2).

\subsection{1. "No tenemos a nadie": rozando la exclusión social}

Estas familias carecen de una red de apoyo potencial o dicha red se encuentra dañada. Por tanto, perciben que no disponen de una red de apoyo, ni formal ni informal, que pueda sostenerlas en caso de necesidad. En este sentido, creen que los únicos recursos a los que pueden acceder de cara a la subsistencia y mejora de sus condiciones socioeconómicas son aquellos de los que dispone el propio hogar y sus miembros. Estas familias resultan especialmente vulnerables ante el deterioro de las condiciones materiales y ante los sucesos desestabilizantes.

\subsection{2. "Nos ayudan como pueden": entre la exclusión y la inclusión}

La red de relaciones de la que disponen estas familias es frágil, pequeña o inestable, por lo que se percibe disponibilidad del apoyo con limitaciones en caso de necesidad. Estas limitaciones se reflejan en las condiciones en las que se configura este apoyo. Las redes familiares y de amistades, como sistema, pueden carecer de la capacidad de protección y de apoyo o tener una capacidad reducida. Las redes formales e institucionales, por otra parte, están sujetas a normas (requisitos de acceso, condiciones, régimen de incompatibilidades) y carecen de la flexibilidad e inmediatez de las redes informales. Los recursos a los que pueden acceder de cara a la subsistencia y mejora de sus condiciones socioeconómicas son aquellos de los que dispone el propio hogar y, aunque no incondicionalmente, aquellos recursos de las redes formales e informales. En este contexto, la integración en redes comunitarias será vital para la diversificación de estrategias resilientes y, en consecuencia, aumentar las posibilidades de mejora.

\subsection{3. "Menos mal que tenemos a... ": manteniéndose en la inclusión}

El hogar cuenta con red de apoyo estable y sólida con capacidad de protección ante la adversidad. Perciben disponibilidad de apoyo en caso de necesidad, ya sea porque cuentan con experiencias previas de apoyo activo por parte de miembros de sus redes, ya porque se perciben plenamente integradas. En este sentido, los recursos a los que pueden acceder de cara a la subsistencia y a la mejora de sus condiciones socioeconómicas son aquellos de los que dispone el propio hogar y aquellos recursos provenientes de las redes formales e informales.

\section{Conclusiones}

El acceso a los recursos económicos y la disponibilidad de apoyos sociales son los dos ejes a partir de los que hemos diseñado un primer modelo de afrontamiento en situaciones de vulnerabilidad social. Si bien existe la convicción de que la percepción subjetiva de apoyo en la familia y la red de amistades es poco sensible a la gradación -es 
decir, se dispone o no se dispone- (Comité Técnico de la Fundación Foessa, 2017) sí que se percibe cierta variabilidad en la percepción sobre las condiciones en las que se da ese apoyo: si es incondicional, o por el contrario, está limitado (en tiempo o alcance, bien debido a la mermada capacidad de las familias y amistades estresadas por la crisis, o debido a la censura autoimpuesta a la hora de solicitar apoyo, causada por el propio estrés añadido, y por los costes emocionales y sociales que conlleva depender de las demás). En esta variabilidad -que introduce cierta gradación a la disponibilidad de apoyo, sobre todo proveniente de la red informal-, inciden dos variables importantes: el tiempo/cronicidad de la situación de vulnerabilidad y la situación del entorno más próximo.

En el primer caso, se aprecia que las familias vulnerables cuya situación se perpetúa en el tiempo y se cronifica parecen agotar los apoyos de familia y amistades, puesto que no es posible mantener dicha tensión durante un periodo largo de tiempo. En el segundo caso, no podemos pasar por alto el hecho de que es posible que familias nucleares vulnerables pertenezcan a familias extensas que vivan una situación similar, o incluso peor, como consecuencia de la desigualdad heredada (Franzini, Raitano y Vona, 2016). En este sentido, debemos tener en cuenta que las condiciones estructurales, que son determinantes en el afrontamiento de situaciones de vulnerabilidad, no son únicamente el escenario donde se desarrolla dicho proceso, sino que son parte del propio proceso de afrontamiento. Este hecho justifica y hace imperativo un sistema de bienestar que sea capaz de proteger y paliar las condiciones sistémicas y estructurales de desigualdad.

La percepción de seguridad es un mecanismo clave en la superación de la vulnerabilidad. Disponer de ingresos regulares suficientes para poder afrontar los gastos relativos a la vivienda -o disponer de una vivienda libre de gastos-y poder garantizar los suministros son dos de los elementos que mayor sensación de seguridad aportan a las familias. En este sentido, cabe destacar el rol jugado por algunas de las prestaciones sociales, como la RGI, que, aunque no está exenta de costes emocionales y sociales para las personas perceptoras (estigma social, vergüenza), se constituye como una red de seguridad fundamental para las personas desempleadas que han agotado sus propios recursos económicos y otro tipo de prestaciones y ayudas institucionales.
La RGI se constata como un factor importante de integración -aunque no suficiente por sí mismo, coincidiendo con Elorrieta (2013) - y garante de mecanismos de afrontamiento. En este punto, merece la pena comentar el hecho de que quizá podría estudiarse la posibilidad de ampliar el perfil de las familias con derecho a la RGI a partir de un análisis no sólo de la situación familiar, sino de la situación individual. Quizá esta prospección ayudaría a eliminar ciertas reticencias y críticas que, a menudo, reciben prestaciones como la RGI, por considerar -falsamente- que tan sólo responden a las necesidades de determinados colectivos sociales.

El paro de larga duración de las personas de mediana edad es uno de los retos a los que se enfrenta el desempleo en Euskadi (Eustat, 2016a), y puesto que afecta a la disponibilidad de apoyos no sólo formales sino también informales, esta cronicidad del desempleo en las personas mayores de 45 años merecería una especial atención y medidas concretas de intervención, atendiendo de manera específica a la situación de las mujeres, cuyo desempleo de larga duración les afecta también en otros momentos de su ciclo vital (Eustat, 2016b).

Por último, pero no menos importante, se ha observado, en la propia obtención de la muestra y en el análisis de los datos sobre personas perceptoras de la RGI, que la vulnerabilidad tiene, frecuentemente, rostro de mujer. La situación vivida por las familias monomarentales y por las mujeres mayores viudas deberían ser ámbitos clave de intervención.

En suma, si atendemos a la disponibilidad de recursos económicos y al apoyo social como ejes de un modelo de afrontamiento ante situaciones de vulnerabilidad, redescubrimos los riesgos derivados de la cronicidad del desempleo, puesto que no sólo desaparecen importantes fuentes de recursos públicos e institucionales, sino también los derivados de apoyos familiares. En ese momento, cobran una importancia significativa algunas asociaciones del tercer sector (Cáritas, Cruz Roja, bancos de alimentos) y comunidades religiosas. Dejamos abierto el interrogante sobre si es precisamente ése el papel que deben jugar estas asociaciones (¿último salvavidas, último y - poco visible- recurso?), o si, por el contrario, el apoyo institucional debería garantizar ámbitos de seguridad también en esos momentos cercanos al desamparo. 
ANAF, J. et al. (2013): “The interplay between structure and agency in shaping the mental health consequences of job loss", BMC Public

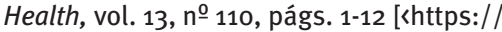
bmcpublichealth.biomedcentral.com/ articles/10.1186/1471-2458-13-110>].

ANDER-EGG, E. (2003): Métodos y técnicas de investigación social IV. Técnicas para la recogida de datos $e$ información, Lumen.

ARISTEGUI, I. et al. (2017): "Vulnerabilidad social percibida en contexto de crisis económica", Revista Española de Sociología, vol. 26, nํㅜ 3 (supl.).

ARISTEGUI, I.; BELOQUI, U.; y MOSTEIRO, A. (2012): “El valor del trabajo", en LEONARDO, J. (coord.), Cambios de valores en los inicios del siglo XXI en Euskadi y Navarra, Bilbao, Universidad de Deusto, págs. 163-190.

AYTAÇ, I.; RANKIN, B.; e IBIKOGLU, A. (2014): “The social impact of the 2008 global economic crisis on neighborhoods, households, and individuals in Turkey", Social Indicators Research, vol. 124, $\mathrm{n}-1$, págs. 1-19.

BUENDÍA, J. (2010): El impacto psicológico del desempleo, Murcia, Universidad de Murcia.

- (1990): “Psicopatología del desempleo”, Anales de Psicología, vol. 16, ํำ1, págs. 21-36.

CHZHEN, Y. (2016): "Perceptions of the economic crisis in Europe: Do adults in households with children feel a greater impact?", Social Indicators Research, vol. 127, n-1, págs. 341-360.

COMITÉ TÉCNICO DE LA FUNDACIÓN FOESSA (2017): "Las redes de protección de los hogares y las familias en la poscrisis", Análisis y Perspectivas, no 6, págs. 3-22 [<https://www. caritasmadrid.org/sites/default/files/Analisis_ perspectivas_2017_caritas.pdf〉].
CORBETTA, P. (2003): Social Research: Theory, Methods and Techniques, Wiltshire, Sage Publications.

CRESWELL, J. (2007): Qualitative Inquiry \& Research Design: Choosing among Five Approaches, Thousand Oaks, Sage Publications.

DAGDEVIREN, H.; DONOGHUE, M.; y MEIER, L. (2016): “The narratives of hardship: The new and the old poor in the aftermath of the 2008 crisis in Europe", The Sociological Review, págs. 1-17 [/https://doi.org/10.1111/1467-954X.12403/].

DAGDEVIREN, H.; DONOGHUE, M.; y PROMBERGER, $M$. (2016): "Resilience, hardship and social conditions", Journal of Social Policy, vol. 45, no 1 1, págs. 1-20 [<https://doi.org/10.1017/ So04727941500032X>].

DUNST, C.; y TRIVETTE, C. (1990): "Assessment of social support in early intervention programs", en MEISELS, S.; y SHONKOFF, J., Handbook of Early Chilhood Intervention, Nueva York, University of Cambridge, págs. 326-349.

EDIS (2010): Redes de apoyo social en las personas en situación de vulnerabilidad y exclusión social en la diócesis de Madrid, Madrid, Cáritas Madrid [<http://www.caritasmadrid.org/sites/ default/files/PDF\%20ESTUDI0\%20DE\%20 REDES\%20DE\%20APOYO\%20SOCIAL.pdf〉].

ELORRIETA, A. (2013): "Zaurgarritasun ibilbideetan balantzaka", Zerbitzuan, no 54 , págs. 31-46 [<https://doi.org/10.5569/1134-7147.54.02)]

ENGEL, R. J.; y SCHUTT, R. K. (2013): The Practice of Research in Social Work, Thousand Oaks, Sage Publications.

EUSTAT (2016a): “Tasa de desempleo de larga duración”, Vitoria-Gasteiz, Eustat-Instituto Vasco de Estadística [rhttp://www.eustat.eus/ 
documentos/opt_o/tema_504/elem_2589/ definicion.html $]$.

- (2016b): “Tasa de paro de larga duración femenina (16 a 74 años) por país. 2007-2016", VitoriaGasteiz, Eustat-Instituto Vasco de Estadística [<http://www.eustat.eus/elem/eleo002500/ tblooo2552_c.html>].

FOLKMAN, S.; y MOSKOWITZ, J. T. (2004): “Coping: pitfalls and promises", Annual Review of Psychology, $n^{\circ}$ 55, págs. $745-774$.

FRANZINI, M.; RAITANO, M.; y VONA, F. (2016): "The channels of intergenerational transmission of inequality: A cross-country comparison", Rivista Italiana degli Economisti, vol. 18, nํㅜ 2, págs. 201-226 [<https://www.rivisteweb.it/ doi/10.1427/73848>].

GOUGH, M.; y KILLEWALD, A. (2011): “Unemployment in families: The case of housework", Journal of Marriage and Family, vol. 73, octubre, págs. 1.085-1.100 [<https://www.ncbi.nlm.nih.gov/ pmc/articles/PMC3207314/>].

HEGELSON, V.; y LOPEZ, L. (2010): “Social support and growth following adversity", en REICH, J.; ZAUTRA, A.; y HALL, J., Handbook of Adult Resilience, Londres, The Guildford Press, págs. 309-330.

ISRAR, S. et al. (2000): “Coping strategies of health personnel during economic crisis: A case study from Cameroon", Tropical Medicine \& International Health, vol. 5, nำ 4, págs. 288 292.

JAHODA, M. (1982): Employment and Unemployment: A Social-psychological Analysis, Cambridge, Cambridge University Press.

JANLERT, U.; y HAMMARSTRÖM, A. (2009): "Which theory is best? Exploratory models of the relationship between unemployment and health", BloMed Central Public Health, vol. 9, no 235 [<https:// www.ncbi.nlm.nih.gov/pmc/articles/ PMC2720386/>].

KALIL, A.; y WIGHTMAN, P. (2011): “Parental job loss and children's educational attainment in black and white middle-class families", Social Science Quarterly, vol. 92, nํ1, págs. 57-78 [<https:// doi.org/10.1111/j.1540-6237.2011.00757.x>].

KROLL, L. E.; y LAMPERT, T. (2011): “Unemployment, social support and health problems", Deutsches Ärzteblatt International, vol. 108, no 4, págs. 47-52 [<https://www.ncbi.nlm.nih.gov/pmc/ articles/PMC3036007/>].

KVALE, S. (1996): Interviews: An Introduction to Qualitative Research Interview, Thousand Oaks, Sage Publications.

KVALE, S.; y BRINKMANN, S. (2015): Interviews: Learning the Craft of Qualitative Research Interviewing, Thousand Oaks, Sage Publications.

LAZARUS, R. S. (1999): Stress and Emotion: A New Synthesis, Nueva York, Springer.

- (1981): “The stress and coping paradigm”, en EDISDORFER, C. et al., Models for Clinical Psychopathology, Nueva York, Spectrum Medical and Scienti Books, págs. 177-214.

- (1966): Psychological Stress and the Coping Process, Nueva York, McGraw-Hill.
LAZARUS, R. S.; y FOLKMAN, S. (1984): Stress, Appraisal, and Coping, Nueva York, Springer.

LAZARUS, R. S.; y LAUNIER, R. (1978): “Stress-related transactions between person and environment", en PERVIN, L. A.; y LEWIS, M., Perspectives in Interactional Psychology, Nueva York, Plenum Press, págs. 287-327.

LEVIN, I.; y TROST, J. (1999): “Living apart together”, Community, Work \& Family, vol. 2, nº 3, págs. 279-294.

LORENZO, F. (coord.) (2014): VII Informe sobre exclusión y desarrollo social en España, Madrid, Fundación FOESSA.

MARTÍN PADILLA, E. et al. (2007): “Estrategias de afrontamiento de crisis causadas por desempleo en familias con hijos adolescentes en Bogotá", Acta Colombiana de Psicología, vol. 10, nํㅜ 2, págs. 127-141 [<http://www. redalyc.org/html/798/79810213/ >].

MARTÍNEZ VIRTO, L. (2017): “La crisis se lleva la capacidad de resistir de muchas familias en España”, Análisis y Perspectivas, nº 6, págs. 24-28 [<https://www.caritasmadrid.org/sites/default/ files/Analisis_perspectivas_2017_caritas.pdf〉].

MENAGHAN, E. G. (1991): "Work experiences and family interaction processes: The long reach of the job?", Annual Review of Sociology, vol. 17, págs. 419-444 [<https://doi.org/10.1146/annurev. so.17.080191.002223〉]

MILES, M.; HUBERMAN, A.; y SALDAÑA, J. (2014): Qualitative Data Analysis: A Methods Sourcebook, Thousand Oaks, Sage Editions.

NEPPL, T. K. et al. (2015): "The impact of economic pressure on parent positivity, parenting, and adolescent positivity into emerging adulthood", Family Relations. Interdisciplinary Journal of Applied Family Studies, vol. 64, págs. 80-92 [<https:// doi.org/10.1111/fare.12098>].

PAÍS VASCO (2017): “Decreto 16/2017, de 17 de enero, de modificación del Decreto de ayudas de emergencia social", Boletín Oficial del País Vasco, no 16, 24-1-17 [<http://www. euskadi.eus/ayuda_subvencion/ayudas-deemergencia-social-aes/webo1-s2enple/es/>]

- (2011): “Ley 4/2011, de 24 de noviembre, de modificación de la Ley para la Garantía de Ingresos y para la Inclusión Social”, Boletín Oficial del País Vasco, nㅇ 233, 12-12-11 [<https://www.euskadi.eus/ y22-bopv/es/bopv2/datos/2011/12/1105924a. $p d f>]$.

- (2011): "Decreto 4/2011, de 18 de enero, de las Ayudas de Emergencia Social", Boletín Oficial del País Vasco, no 27, 9-2-11 [<http://www.euskadi.eus/ ayuda_subvencion/ayudas-de-emergenciasocial-aes/webo1-s2enple/es/>].

- (2010): “Decreto 2/2010, de 12 de enero, de la prestación complementaria de vivienda", Boletín Oficial del País Vasco, no 11, 19-1-10 [<http://www. ivap.euskadi.eus/r61-vedorok/es/contenidos/ decreto/bopv201000216/es_def/index.shtml>].

- (2010): “Decreto 147/2010, de 25 de mayo, de la Renta de Garantía de Ingresos", Boletín Oficial del País Vasco, nํ1시 17-6-10 [<https://www.euskadi. eus/bopv2/datos/2010/o6/1003020a.pdf>]. 
- (2008): "Ley 18/2008, de 23 de diciembre, para la Garantía de Ingresos y para la Inclusión Social", Boletín Oficial del País Vasco, no 250, 31-12-08 [rhttps://www.euskadi.eus/r47-bopvvaci/es/ bopv2/datos/2008/12/0807235a.shtml>].

PATTERSON, J. M. (2002a): "Integrating family resilience and family stress theory", Journal of Marriage and Family, vol. 64, mayo, págs. 349-360 [<https:// doi.org/10.1111/j.1741-3737.2002.00349.x〉].

- (2002b): “Understanding family resilience”, Journal of Clinical Psychology, vol. 58, noำ págs. 233-246 [/https://doi.org/10.1002/jclp.10019)].

REVILLA, J.; MARTÍN, P.; y DE CASTRO, C. (2017): “The reconstruction of resilience as a social and collective phenomenon: Poverty and coping capacity during the economic crisis", European Societies ['https://doi.org/10.1080/14616696. 2017.1346195>].

REQUENA, F. (2011): “La importancia de las redes de apoyo", en REQUENA, F., Las redes de apoyo social, Cizur Menor, Thompson Reuters, págs. 17-29.

RUIZ OLABUÉNAGA, J. (2012a): Metodología de la investigación cualitativa, Bilbao, Universidad de Deusto.

- (2012b): Teoría y práctica de la investigación cualitativa, Bilbao, Universidad de Deusto.

RUIZ OLABUÉNAGA, J.; e ISPIZUA, M. (1989): La descodificación de la vida cotidiana, Bilbao, Universidad de Deusto.

SACCHETO, D.; y VIANELLO, F. (2015): “Unemployed migrants coping with the economic crisis: Romanians and Moroccoans in Italy", Journal of Internation Migration and Integration, vol. 17, n⿳0 3, págs. 839-852.
SALDAÑA, J. (2016): The Coding Manual for Qualitative Researchers, Londres, Sage Publications.

SCHLIEBNER, C. T.; y PEREGOY, J. J. (1994): "Unemployment effects on the family and the child: Interventions for counselors", Journal of Counseling \& Development, vol. 72, págs. 368-372 [<https://doi. org/10.1002/j.1556-6676.1994.tboo951.x>].

UNIVERSIDAD DE DEUSTO (2013-2017): DeustoBarómetro Social [khttp://www.barometrosocial.deusto. es>].

VILA, D. (2014): “Vida familiar y condiciones de vulnerabilidad. Aproximación a la situación de vulnerabilidad de las familias monoparentales en España", en BARRANCO, M.; y CHURRUCA, C., Vulnerabilidad y protección de los Derechos Humanos, Valencia, Tirant lo Blanch, págs. 249-276.

VILLARDÓN, L. et al. (2011): Informe Bizkailab 2011. El desempleo como factor de vulnerabilidad a la exclusión social: el proyecto de vida como factor de protección, Bilbao, Bizkailab [khttps://www.bizkailab.deusto.es/wpcontent/uploads/2012/04/5707-Report-01Inserci\%C3\%B3n-laboral.pdf $>$ ].

WECKSTRÖM, S. (2012): “Self-assessed consequences of unemployment on individual wellbeing and family relationships: A study of unemployed women and men in Finland", International Journal of Social Welfare, vol. 21, págs. 372-383 [khttps://doi.org/10.1111 /j.1468-2397.2011.00829.x>].

WERNER, E.; y SMITH, R. (1992): Vulnerable but Invincible: A Longitudinal Study of Resilient Children and Youth, Nueva York, McGraw Hill Book. 